\title{
Effect of dual endothelin receptor blockade on ocular blood flow in patients with glaucoma and healthy subjects
}

\author{
Michael Lasta ${ }^{1}$, Hemma Resch ${ }^{1,2}$, Katharina Karl ${ }^{1}$, Günther Weigert ${ }^{1,2}$, \\ Michael Wolzt ${ }^{1}$, Anton Hommer ${ }^{1}$, Leopold Schmetterer*1,3 and \\ Gerhard Garhöfer ${ }^{1}$
}

Address: ${ }^{1}$ Department of Clinical Pharmacology, Medical University of Vienna, 1090 Vienna, Austria, ${ }^{2}$ Department of Ophthalmology, Medical University of Vienna, 1090 Vienna, Austria and ${ }^{3}$ Center for Biomedical Engineering and Physics, Medical University of Vienna, 1090 Vienna, Austria

Email: Leopold Schmetterer* - leopold.schmetterer@meduniwien.ac.at

* Corresponding author

from 14th Scientific Symposium of the Austrian Pharmacological Society (APHAR)

Innsbruck, Austria. 21-22 November 2008

Published: 5 November 2008

BMC Pharmacology 2008, 8(Suppl I):A50 doi:10.II86/I47I-22I0-8-SI-A50

This abstract is available from: http://www.biomedcentral.com/I47I-22I0/8/SI/A50

C 2008 Lasta et al; licensee BioMed Central Ltd.

\section{Background}

Several lines of evidence indicate that altered blood flow regulation may contribute to the pathogenesis of glaucomatous optic neuropathy. Recent data support the hypothesis that the endothelin system is involved in the processes that leads to vascular dysregulation in glaucoma. Here, we tested the hypothesis that bosentan, a dual endothelin receptor antagonist, increases ocular blood flow in patients with glaucoma.

\section{Materials and methods}

Fourteen patients with primary open-angle glaucoma and 14 sex and age-matched healthy volunteers were included. Both groups received bosentan $500 \mathrm{mg}$ daily for 8 days. Ocular hemodynamics were assessed at baseline and on the last study day. Retinal vessel diameters and retinal red blood cell velocity were recorded with a Retinal Vessel Analyser and laser Doppler velocimetry, respectively. Choroidal and optic nerve head blood flow were measured with laser Doppler flowmetry.

\section{Results}

Retinal arteries and veins showed a significant dilatation after administration of bosentan in both groups $(+5 \%$ to $8 \%$ ). Retinal blood velocity and retinal blood flow increased up to $+45 \%$ after administration of bosentan in both patients and healthy subjects. Administration of bosentan increased choroidal $(+12 \%$ to $17 \%)$ and optic nerve head blood flow $(+11 \%$ to $24 \%)$ in both groups. The effect of bosentan on ocular blood flow parameters was comparable between the two groups.

\section{Conclusion}

The data of the present study indicate that dual inhibition of endothelin receptors increases ocular blood flow in patients with glaucoma and healthy subjects. Further studies are required to study the dose-response relationship of this effect and to characterize the role of endothelin receptor subtypes. 